\title{
18
}

\section{EVALUACIÓN DE LAS TOPOLOGÍAS FÍSICAS DE REDES WSN PARA LA MEDICIÓN DE VARIABLES AMBIENTALES.}

S.F. Saigua Carvajal, M.V. Villafuerte Haro, D. Ávila-Pesantez, A.L. Arellano Aucancela 


\title{
EVALUACIÓN DE LAS TOPOLOGÍAS FÍSICAS DE REDES WSN PARA LA MEDICIÓN DE VARIABLES AMBIENTALES.
}

\author{
S.F. Saigua Carvajal ${ }^{1}$, M.V. Villafuerte $\operatorname{Haro}^{1}$, D. Avila-Pesantez ${ }^{1}$, A.L. Arellano Aucancela ${ }^{1}$ \\ ${ }^{1}$ Escuela de Ingeniería en Electrónica, Telecomunicaciones y Redes, Facultad de Informática y Electrónica, Escuela \\ Superior Politécnica de Chimborazo (ESPOCH) \\ Riobamba - Ecuador \\ E-mail: ssaiga@espoch.edu.ec
}

\begin{abstract}
Resumen
En este trabajo se presenta un estudio comparativo entre las topologías físicas que apoyan WSN con el fin de determinar la más eficaz aplicado a una red inalámbrica de sensores ambientales. La investigación se realizó mediante el apoyo del Network Simulator 2 (NS-2), que permite crear un entorno similar al real y simulado su funcionamiento, para determinar la mejor topología de un método inductivo se aplicó para evaluar los datos de NS-2 que se basaron en las métricas de rendimiento, tales como: el envío de paquetes, el consumo de energía y la cobertura. Como resultado se obtuvo que la topología física estrella es la mejor manera de aplicar una red WSN para las mediciones ambientales, que tiene una relación de Entrega de paquetes del 97,9\%, el rendimiento de 0,7542 Kbps, un retraso de 0,0162 ms, un consumo de energía bajo y una mayor área de cobertura del sensor.
\end{abstract}

Palabras clave: Wireless Sensor Network, Relación de entrega de paquetes, sensor digital, topologías WSN

\begin{abstract}
This paper presents a comparative study between physical topologies that support WSN in order to determine the most efficient applied to a wireless network of environmental sensors. The research was performed by the support of Network Simulator 2 (NS-2), it allows to create an environment similar to real and simulated its operation, to determine the best topology an inductive method was applied to evaluate the data from NS-2 that were based on the performance metrics such as: sending packages, energy consumption and coverage. As a result it was obtained that the star physical topology is the best to implement a WSN network for environmental measurements, that has Packet Delivery Ratio of 97,9\%, Throughput of 0,7542 Kbps, a delay of 0,0162 ms, a low energy consumption and a greater sensor coverage area.
\end{abstract}

Keywords: Wireless Sensor Network, Packet Delivery Ratio, Digital sensor, WSN topologies. 


\section{Introducción}

En el Ecuador obtener información sobre los fenómenos medioambientales es muy importante ya que debido a la ubicación (latitud $0^{\circ}$ ) tienen un comportamiento diferente a los estudios realizados en otros países [1]. Uno de los problemas que dificulta el desarrollo de estos estudios surge debido a la monitorización de manera rústica de las estaciones meteorológicas, y por lo general, necesitan de una persona que acuda hasta el sitio para extraer la información recolectada. Por otra parte, las estaciones que han evolucionado utilizan sistemas de radio enlaces o GPRS como alternativa para solucionar este problema, pero los gastos son significativos cuando cubren poca distancia.

Debido a esta problemática surge la idea de realizar un estudio comparativo de las topologías físicas en estrella y árbol de redes Wireless Sensor Network (WSN) y determinar cuál es la más adecuada para evolucionar la forma de recolección de datos al brindar un sistema de comunicación inalámbrico a bajo costo que permita monitorear distintas variables ambientales en tiempo real. Para ello se utilizará el software Network Simulator (NS-2) [2], en código abierto, que permite crear un ambiente similar al real y analizar su funcionamiento en ambas topologías físicas. Para determinar los resultados, se realizará un análisis comparativo teniendo como métricas de evaluación: pruebas de envío de paquetes, consumo de energía y cobertura [3].

\section{Metodología}

\subsection{Materiales}

Los materiales utilizados fueron:

- $\quad$ Software NS-2

- $\quad$ Software Minitab v.16

- Delphi 7

- 4 Micro-Controladores Arduino Uno

- 5 Módulos de comunicación XBee

- 1 Módulo XBee USB explorer

- 4 Digital Humidity Temperature Sensor (DHT11).

\subsection{Desarrollo}

El escenario fue desarrollado en NS-2, que permite configurar la pila de protocolos de comunicación mediante un script y otros aspectos básicos del tipo de red a simular. Conforme avanza la simulación, se generan un conjunto de datos de salida que se almacenan en un archivo de traza cuya extensión es .tr. A partir de las trazas se utiliza el lenguaje AWK para filtrar la información que se requiere. Finalmente, la herramienta Network Animator (archivos con extensión .nam) permite realizar un análisis visual del envío y recepción de paquetes de datos y control.

Para el estudio se tomó en cuenta las variables más relevantes en análisis de eficiencia y consumo de energía de las topologías de una red WSN [4]. Para dicho efecto se analizan los siguientes aspectos:

- Paquetes enviados, recibidos, rechazados y reenviados en la red.

- Retardo de los paquetes que son enviados por los nodos finales al nodo coordinador.

- Throughput de la red WSN.

- Energía consumida por la red en total.

- Cobertura.

\section{A.- Configuración del simulador}

a) Características de la red WSN

Son variables creadas por el usuario caracterizar la simulación. En este caso hay que redefinir las siguientes: [4]

- $\quad$ Tipo de canal= WirelessChannel

- Modelo de radio Propagación= TwoRayGround

- $\quad$ Tipo de capa de enlace $=$ LL

- Modelo de antena= OmniAntenna

- Número de nodos= 11

- Protocolo de enrutamiento= Ad Hoc On-Demand Vector Routing (AODV).

- $\quad$ Tipo de Trafico= UDP/CBR

- Modelo de energía= EnergyModel

b) Configuración de los nodos

Se especifica una configuración general indicando la posición y el comportamiento de cada uno de ellos, ya sean nodos finales, enrutadores o coordinadores.

c) Creación de los agentes de tráfico

Los nodos por si solos no generan ni reciben tráfico, para hacerlo hay que crear los agentes. Una vez creados estos agentes se deben asociar a distintos nodos, de estos agentes dependerán los protocolos de las capas de transporte y superiores. Se creó agentes UDP ya que simulan al tráfico generado por una WSN.

\section{B.- Ejecución de la simulación}

Para el escenario se construyó una red con 1 nodo coordinador y 10 nodos finales, y se definió dos 
topologías: estrella y árbol, como se visualiza en la Figura 1.

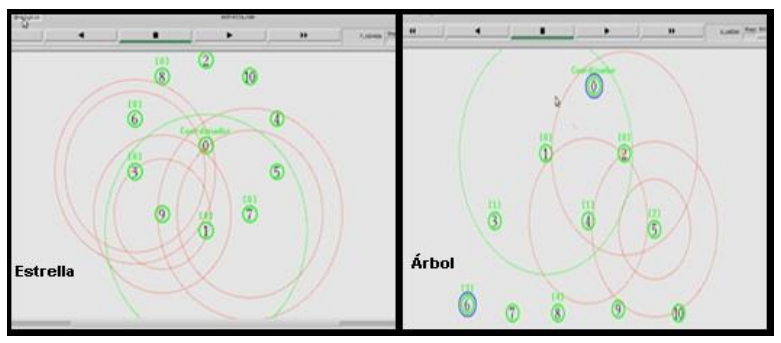

Fig. 1 Resultado del archivo .nam para la topología estrella y árbol.

Se decidió utilizar las siguientes variables estadísticas [5]:

- Packet Delivery Ratio (PDR): Es la relación entre el número de paquete de datos entregado al destino.

$$
\text { POR }=\frac{\sum \text { Paquates rocibidos }}{\sum \text { Paqutes anviados }}
$$

- Throughput: Se llama así al volumen de trabajo o de información que fluye a través de un sistema, en un determinado tiempo, en este caso tiempo de simulación.

$$
\text { Randimian to }=\frac{\text { Númaro de bits }}{\text { Tigmpo de simulación }}
$$

- End-to-End Delay: Es el tiempo que necesita un paquete de datos para llegar a su destino. También incluye la demora causada por el proceso de descubrimiento de ruta y la cola en la transmisión de paquetes de datos.

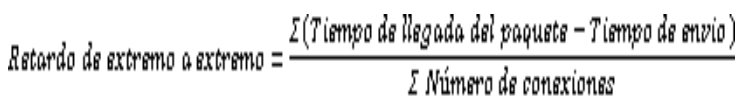

- Consumo de Energía: Se realiza con una simple resta del consumo de energía en cada suceso de la red.

Con estas fórmulas establecidas en las ecuaciones (1), (2) y (3), se puede observar los datos generados por la simulación en el archivo AWK para la topología estrella y los datos de la topología árbol. Véase la Figura 2 y Figura 3 respectivamente.

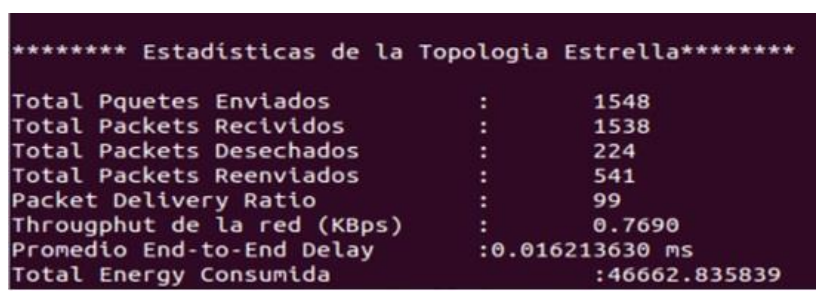

Fig. 2 Datos Estadísticos topología estrella.

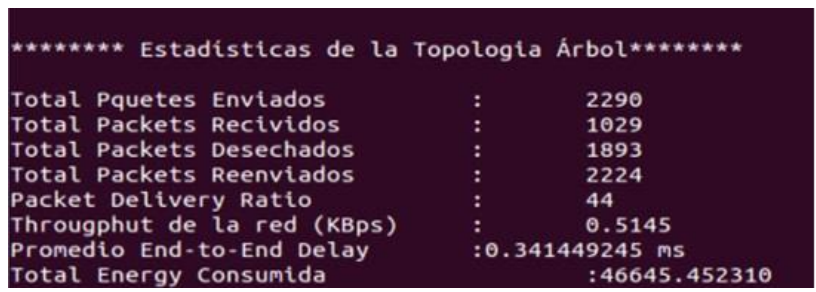

Fig. 3 Datos Estadísticos topología árbol.

\section{- Área de Cobertura}

Es el área donde se toman los datos de los sensores, y esto depende del tipo de sensores con los que se trabaje, en nuestro escenario planteado se utilizó el sensor DHT11, que tiene una conexión de hasta 20 metros a la unidad de procesamiento. [6]. Para calcular el área se realiza las siguientes operaciones:

Área ${ }_{\text {sensor/nodo }}=\Pi \mathrm{r}^{2}$

Área ${ }_{\text {sensor/nodo }}=\Pi(20)^{2}$

Área ${ }_{\text {sensor } / \text { nodo }}=1256,64 \mathrm{~m}^{2}$

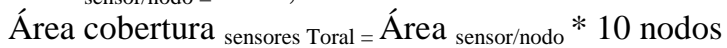

Área cobertura ${ }_{\text {sensores Toral }}=1256,64 * 10=12566,4 \mathrm{~m}^{2}$

El escenario de la topología árbol consta de 1 nodo coordinador, 5 nodos enrutadores y 5 nodos finales, (Véase figura 4) donde se va a calcular el área de cobertura:

Área ${ }_{\text {sensor } / \text { nodo }}=\Pi r^{2}$

Área ${ }_{\text {sensor/nodo }=\Pi(20)^{2}}$

Área ${ }_{\text {sensor } / \text { nodo }}=1256,64 \mathrm{~m}^{2}$

Área cobertura sensores Toral $=$ Área sensor/nodo $^{*} 5$ nodos

Área cobertura ${ }_{\text {sensores Toral }}=1256,64 * 5=6238,19 \mathrm{~m}^{2}$

Se concluye que la topología estrella tiene una mayor área de medición de datos, mientras que la topología árbol desperdicia recursos, ya que, los nodos enrutadores no toman ningún tipo de medición y únicamente pasan la información de los nodos finales al nodo coordinador. Por tanto, la topología estrella tiene un mayor área de cobertura de sensado. 


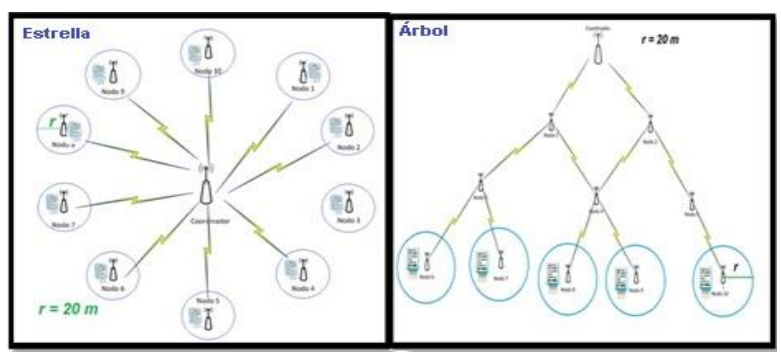

Fig. 4 Área de cobertura de las topologías Estrella y Árbol.

\section{C.- Diseño del prototipo}

Se diseñó el prototipo en laboratorio, que cuenta con cuatro nodos finales y un nodo coordinador tal como lo podemos observar en la Figura 5.

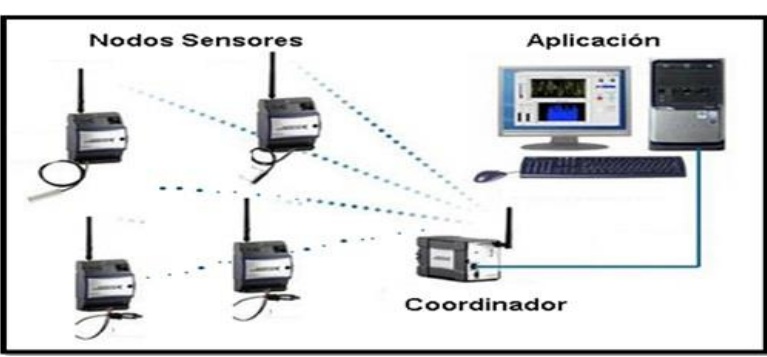

Fig. 5 Diseño del prototipo con la topología Estrella

Cada uno de los nodos recogió información del medioambiente a través del sensor DHT11, que está conectado a la placa base, la cual procesó la información y le agregó una cabecera a los datos para identificar de que nodo proviene. Una PC conectada con una aplicación permite a los usuarios ver el funcionamiento de la red WSN. [7], [8].

\section{Dispositivos Finales (Nodos)}

El nodo final está formado por cuatro componentes básicos: un sensor DHT11; una unidad de proceso (Arduino Uno) [9]; una unidad del transmisor-receptor (XBee s1); y una unidad de potencia (batería de 9V), tal como lo vemos en la Figura 6.

\section{Dispositivo Coordinador}

El nodo coordinador está formado por un módulo de comunicación XBee S1, el cual se conecta directamente a la computadora utilizando un XBee USB Explorer.

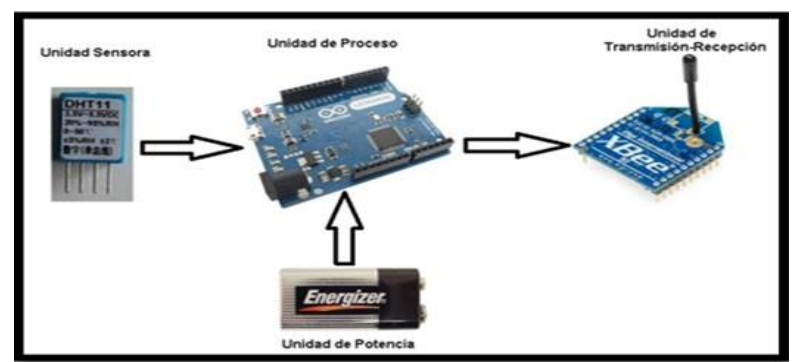

Fig. 6 Componentes de un Nodo Sensor.

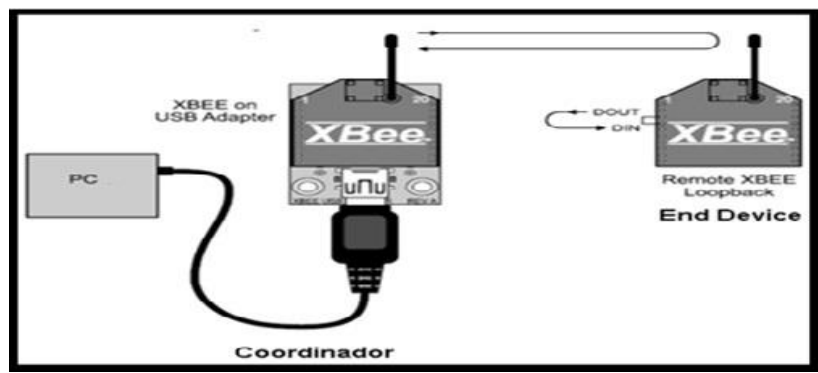

Fig. 7 Componentes Nodo Coordinador XBee.

\section{Aplicación}

Una vez que el nodo coordinador envía los datos a la PC, la aplicación programada en Delphi 7 con una interfaz gráfica amigable, almacena la información en la Base de datos MySQL [10]. Además, muestra las gráficas en tiempo real de los valores capturados por cada uno de los nodos. (véase figura 8).

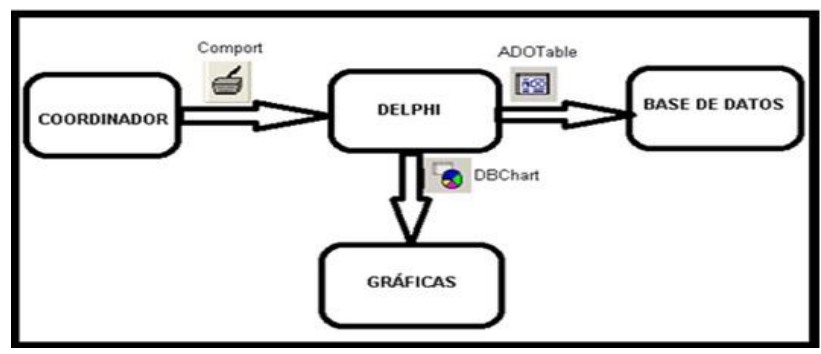

Fig. 8 Esquema de comunicación en la Base de Datos.

\section{Resultados y Discusión}

Para la evaluación, se realizó 20 tomas de muestras (archivos AWK) de cada parámetro establecido utilizando la topología de estrella y topología de árbol, luego, se empleó el método estadístico de diseño completamente aleatorio (DCA) [11]. Este método sirvió para determinar cómo se comportan el conjunto 
de condiciones experimentales que deben imponerse a una unidad experimental dentro de la topología estrella y árbol. Como resultado se puede obtener dos opciones: que sean iguales o uno de las topologías, sea estadísticamente superior a la otra. Además, se utilizó el software Minitab v.16 [12] para cálculos estadísticos de la media de los 5 parámetros establecidos, luego se efectuó el análisis de varianza, con un nivel de confianza del $95 \%$. Para obtener los resultados finales se utilizó ANOVA. En la Tabla 1, se visualiza los valores resultantes de los parámetros en ambas topologías.

Tabla 1 Resultados calculados para cada parámetro de comparación.

\begin{tabular}{|c|c|c|}
\hline Parámetros & Topología Estrella & Topología Árbol \\
\hline PDR (\%) & $\checkmark_{97,9}$ & 43,95 \\
\hline Rendimiento(Kbps) & $\checkmark_{0,7542}$ & 0,5147 \\
\hline $\begin{array}{c}\text { Retado extremo a } \\
\text { extremo (ms) }\end{array}$ & $\checkmark_{0,0162}$ & 0,3416 \\
\hline Consumo de Energía (J) & 46662,17 & $\checkmark_{46645,58}$ \\
\hline $\begin{array}{c}\text { Área de cobertura de } \\
\text { sensado }\left(\mathrm{m}^{2}\right)\end{array}$ & $\checkmark_{12566,4}$ & 6283,2 \\
\hline
\end{tabular}

Una vez analizados cada uno de los parámetros se ha marcado los campos con un $\checkmark$, indicando la métrica con el mejor desempeño. Los resultados obtenidos son:

- La relación de distribución de paquetes (PDR) de la topología estrella es más eficiente que en la topología árbol, lo que nos indica que un $97,9 \%$ de los paquetes enviados, llegan con éxito a su destino.

- El rendimiento en la topología estrella es superior a la topología árbol, esto nos indica que en la topología estrella fluye mayor cantidad de información en un tiempo determinado.

- El retardo extremo a extremo en la topología estrella es menor que en la topología árbol, lo que nos indica que toma menos tiempo enviar un paquete a su destino utilizando la topología estrella.

- El consumo de energía en la topología estrella es mayor que el consumo en la topología árbol, como se busca que las redes WSN consuman la menor cantidad de energía decimos que la topología árbol es la más eficiente.

- El área de cobertura de sensado en la topología estrella es superior a la cobertura de la topología árbol, por tanto decimos que la topología estrella es la más adecuada para obtener mayor cantidad de información.

\section{Conclusiones}

Al evaluar el desempeño y calidad de la Red WSN mediante métricas de evaluación, utilizando el simulador NS-2, se obtuvo como resultado que la topología física en estrella, es la más adecuada para implementar una red inalámbrica de sensores para medir variables de temperatura y humedad en entonos ambientales, ya que cuenta con un PDR del 97,9\%, rendimiento de 0,75 Kbps, un retardo de extremo a extremo de 0,016 ms, mayor área de cobertura y un consumo de energía similar en las ambas topologías estudiadas.

Las redes WSN son una opción de solución frente a las tradicionales redes WiFi y celular, debido a sus bajos precios y al bajo consumo energético. Los módulos de comunicación XBEE S1 son adecuados para implementar una red WSN con topología estrella, y cuando los nodos finales se encuentran hasta 100 metros de distancia, si se requiere mayor distancia con la misma topología se debe optar por módulos de la XBEE serie pro 2 que pueden llegar hasta $7 \mathrm{~km}$.

\section{Referencias Bibliográficas}

[1] Rengel, D. S. (2009). Bienes y servicios ambientales, una herramienta para la conservación de los recursos naturales en el Ecuador.

[2] The Network Simulator - NS-2. [Web en línea]., < http://www.isi.edu/nsnam/ns/ >., [Consulta 2014-10-16].

[3] Fidalgo, A.L., González, L.L., Brandariz, J. S. \& González, X. C. (2010). Redes de Sensores sin Cables para Agricultura de Precisión en Regiones Minifundistas.

[4] Wang, N., Zhang, N. \& Wang, M. (2006). Wireless sensors in agriculture and food industry recent development and future perspective. Computers and Electronics in Agriculture.

[5] Arias, D. A. \& Gudiño, D. S. (2007). Estudio, diseño e implementación de una red sensorial inalámbrica para el monitoreo y control de temperatura en un ambiente industrial reducido.

[6] DHT11 sensor. [web en linea]., < http://panamahitek.com/dht11sensor-de-humedadtemperatura-para-arduino/ >, [Consulta 201501-12].

[7] Díaz, M. (2007). Wireless Sensor Networks: Estado del Arte e Investigación. Med 5-12

[8] Faludi, R. (2010). Building Wireless Sensor Networks., 1a ed., New York-Estados Unidos., Ed. BRIAN. 320p.

[9] MANUAL DE ARDUINO [web en linea]., < http://rua.ua.es/dspace/bitstream/10045/11833/1/arduino.pdf $>$ [Consulta 2014-10-16].

[10] Ahmad, K., (2008). Automation in Construction, Wireless Sensor Networks as part of a Web-Based Building Environmental Monitoring System., Maryland- Estados Unidos., Pp 729-736.

[11] Cochran, W. \& Cox, G. (1965). Diseños experimentales. ed. México.

[12] MINITAB. [Web en línea]., < http://www.minitab.com/enus/Press-Releases/Minitab-16-Opens-the-Door-to-QualityImprovement// >., [Consulta 2014-10-16]. 